\title{
A comparative study on detecting bacterial flora of the nasal cavity in normal healthy workers that work in cement factory and the healthy Students in Koya University
}

\author{
Srwa A. Muhammed ${ }^{1}$, Abdullah W. Mohammed ${ }^{2}$, Awat S. Wadi ${ }^{3}$, Dereh L. \\ Mohammed $^{4}$ \\ ${ }^{1}$ (Biology Department, Faculty of Science\& Health, University of Koya, Iraq/ Kurdistan) \\ ${ }^{2}$ (Biology Department, Faculty of Science\& Health, University of Koya, Iraq/ Kurdistan) \\ 3 ( teacher at Daroxa School, Iraq/Sulaimania) \\ ${ }^{4}$ (Junior resident doctor at Teaching Hospital, Iraq/Sulaimania)
}

\begin{abstract}
Summary: In this study, a total of 45 samples were collected from the nasal cavity of biology healthy students that taking education in the 2012-2013 in Koya University, Faculty of Science and health, and 35 healthy workers that work in Mas cement factory were attending in this study.

From this 45 healthy students seventeen (37.77\%) of them had no growth isolated, sixteen of them $(35.55 \%)$ were found out that they had Streptococcus spp. While nine (20\%) students had Staphylococcus spp. two (4.44\%) of the isolates were E. coli and two (4.44\%) of the isolates overall these 45 students were Klebsiella spp.

From 35 healthy workers were attending in this study, two (5.71\%) of the workers were found that they had no growth isolated, while eighteen (51.42\%) of the workers were found out that they had Moraxella catarrhalis, thirteen (37.14\%) of the workers had Candida albicans, five of them (14.28\%) had streptococcus faecalis, three (8.57\%) of them had E.coli, two (5.71\%) of them had Klebsiella spp., and one (2.85\%) of the growth found Citrobacter freundii.

The nasal Streptococcus spp. Isolates were found out significantly that were higher than other isolates.

The nasal Moraxella catarrhalis Isolates were found out significantly higher than other isolates among the workers that have contact with cement.
\end{abstract}

Key word: Candida albicans, Moraxella catarrhalis, nasal swab, Staphylococcus aureus, Streptococcus pyogenes.

\section{Introduction}

Nasal cavity a large air filled space, main function is to keep foreign or unwanted particles from entering the lungs through the nasal cavity. The nares environment is colonized by a temporally stable microbiota that is distinct from other regions of the integument. Negative association between S. aureus, S. epidermidis, and other groups suggests microbial competition during colonization of the nares, a finding that could be exploited to limit S. aureus colonization. [1]

Recent evidence strongly suggests that the microbiota of the nasal cavity plays a crucial role in determining the reaction patterns of the mucosal and systemic immune system. However, little is known about the normal microbiota of the nasal cavity. [2]

Bioaerosol concentrations at $50 \mathrm{~m}$ upwind of site operations were within a range considered to be 'typical' background levels, with the large majority $(84 \%+)$ of samples yielding less than $1,000 \mathrm{cfu} / \mathrm{m}^{3}$ air of bacteria, actinomycetes, fungi and Aspergillus fumigatus. Close to compost handling activities, if workers are not protected from exposure, they may be exposed to concentrations of airborne bacteria and fungi that frequently exceed [3].

A diverse microbial flora is associated with the skin and mucous membranes of every human being from shortly after birth until death. The human body, which contains about $10^{13}$ cells, routinely harbors about $10^{14}$ bacteria. This bacterial population constitutes the normal microbial flora. The normal microbial flora is relatively stable, with specific genera populating various body regions during particular periods in an individual's life. Microorganisms of the normal flora may aid the host (by competing for microenvironments more effectively than such pathogens as Salmonella spp or by producing nutrients the host can use), may harm the host (by causing dental caries, abscesses, or other infectious diseases), or may exist as commensals (inhabiting the host for long periods without causing detectable harm or benefit). Even though most elements of the normal microbial flora inhabiting the human skin, nails, eyes, oropharynx, genitalia, and gastrointestinal tract are harmless in healthy individuals, these organisms frequently cause disease in compromised hosts. 
Viruses and parasites are not considered members of the normal microbial flora by most investigators because they are not commensals and do not aid the host [4].

The bacterial flora of the nasal cavity covers potential bacterial pathogens (PBPs) including Staphylococcus aureus. Several factors, such as diabetes mellitus, intravenous drug abuse, and dialysis treatment, increase the rate of colonization. The reservoir for Staphylococcus aureus skin carriage is the anterior nares. Staphylococcus aureus is considered to be the most important nasal PBP and has been found in $20 \%$ to $25 \%$ of healthy American adults. It was estimated that $30 \%$ of these patients were infected with Staphylococcus aureus and that the patient's nose was its place of origin. Neisseria meningitidis, Moraxella catarrhalis, Streptococcus pneumonia, Haemophilus influenzae are the other PBPs found in the nasal cavity. Carriers of nasal methicillin resistant Staphylococcus aureus (MRSA) are particularly problematic nasal MRSA was detected in $46 \%$ of patients with liver cirrhosis [5].

Streptococci are bacterial disease factors which are not evaluated among nosocomial factors but commonly take place in the nature and are most often met especially in childhood with the throat infections). Especially the Group B streptococci become colonized in pharynx and vagina flora and so can lead to many maternal and neonatal infections such as intra-amniotic infection, early membrane rupture, premature birth risk,

Moraxella species are parasites of the mucous membranes of humans and other warm-blooded animals. $\mathrm{M}$ catarrhalis organisms are cocci that morphologically resemble Neisseria cells. Other relevant characteristics are presented in. M catarrhalis was formerly placed in the genus Neisseria; however, studies of DNA base content, fatty acid composition, and genetic transformation showed that this organism did not belong in that genus. $\mathrm{M}$ catarrhalis is a member of the normal flora in $40-50 \%$ of normal school children; however, it should be considered more than a harmless commensal of the mucous membranes of humans. It is an infrequent, yet significant, cause of severe systemic infections such as pneumonia, meningitis, and endocarditis. It is an important cause of lower respiratory tract infections in adults with chronic lung disease and a common cause of otitis media, sinusitis, and conjunctivitis in otherwise healthy children and adults. $\mathrm{M}$ catarrhalis may cause clinical syndromes indistinguishable from those caused by gonococci, and so it is important to distinguish these organisms from one another. Many strains produce B-lactamase [7].

Candida albicans is widely distributed throughout the human population as both a commensal organism and as an intermittent pathogen. Limited numbers of viable organisms can be cultured from the oropharynx and from the vaginal cavity of normal individuals. However, as a consequence of microbial imbalance and/or transient immunosuppression, C. albicansovergrowth can readily occur to cause mucosal infections, including oral candidiasis (thrush) or vaginal candidiasis [8].

Aims of our study are:

1- Collection the samples from nose of 45 healthy students were taking education in the 2012-2013 in Koya University, School of Science and Health, Department of Biology and Medical Microbiology and Collection from nasal cavity of healthy cement workers.

2- Isolation and identification these microorganisms that found in the nasal cavity of healthy cement workers and healthy students by cultural, morphological and biochemical tests.

3- Study the resistance rat of these microorganisms to different antimicrobial agents.

\section{Materials and Methods}

\section{II.1Design and specimen collection}

A total of 45 Biological students from level two and level four taking education in the 2012-2013 term in Koya University, School of Science and Health, Department of Biology and Medical Microbiology and 35 healthy workers that work in Mas cement factory were taken. Nose samples of students and workers were examined by taking nose swab from the students not having clinical symptoms (pharyngitis, cryptic tonsillitis, sinusitis, otitis media, and other upper respiratory illnesses), the nose swab samples were taken from the anterior nares of nose with the swab soaked with sterile saline and Washing hands thoroughly, Open swab packaging, checking expiry date then remove swab from packaging, Insert the swab into the anterior nare (nostril). Repeat the procedure with the same swab in the other nare. Without contaminating swab, place in the culture medium provided [9].

\section{II.2 Isolation and identification of bacteria}

The nose swab samples was arrived to the laboratory and planted to $5 \%$ sheep blood agar and $7.5 \%$ salt and mannitol culture, Mackony agar and Choclate agar then incubated in the sterilizer of $37^{\circ} \mathrm{C}$.

Gram positive coccus were identified microscopically according to their appearances. The betahemolysis, colony morphology, mannitole fermentation, (coagulase, catalase and DNAase) positive colonies were identified as S.aureus as mentioned befor by [10]. Since most other species of staphylococci do not produce coagulase, it is useful to divide staphylococci into coagulase-positive and coagulase-negative species. 
Coagulase-negative staphylococci are not highly virulent but are an important cause of infections in certain high-risk groups [10].

Another isolates streptococci are part of normal human microbiota, found on the skin, in the upper respiratory tract, digestive tract and oral cavity. Gram stain character of Streptococci is Gram-positive cocci which grow primarily in pairs and in chains of cells. Strepococci are often grouped by hemolytic reaction on blood agar and by serological typing using the Lancefield classification system.

The most significant pathogens are Streptococcus pyogenes (Lancefield Group A), Streptococcus agalactiae (Lancefield Group B) and Streptococcus pneumonia do not belong to lancefield group. Some streptococci (formerly group D) now placed in the genus Enterococcus are of medical significance because they are often difficult to treat because of antibiotic resistance. Hemolytic is a very important feature used to identify the streptococci [11].

Moraxella catarrhalis is a fastidious organisms that do not ferment carbohydrates, nonmotile, Gramnegative, aerobic, oxidase positive diplococcus are non motile that can cause infections of the respiratory system, middle ear, eye, central nervous system, and joints of humans. The most important pathogen in the genus is Moraxella (formerly, Branhamella) catarrhalis [7].

Candida albicans Colonies on Sabouraud dextrose agar at $25^{\circ} \mathrm{C}$ are white to cream, soft, and smooth to wrinkle. This isolate grows at $42^{\circ} \mathrm{C}$ and on media containing cycloheximide [12].

\section{II.3Biochemical tests}

The biochemical tests of suspected bacteria specially for gram negative bacteria that isolated from student nasal cavity and cement workers were detected by IMVC (Indole, methyl red (MR), vogesproskauer (VP) and citrate), triple sugar iron agar (TSI agar), urease, gelatinase, oxidase, catalase test, motility test, esculine test, fermentation of glucose [13].

\section{II.4 Maintenance of bacterial isolates}

The colonies were studied. It must be inoculated on nutrient agar slants, after incubation at $37^{\circ} \mathrm{C}$ for 24 hr. stored at $4^{\circ} \mathrm{C}$, till other bacteriological tests done [14].

Also the isolates were maintained by inoculating a single colony on nutrient agar by streaking method and incubated at $37^{\circ} \mathrm{C}$ for 24 hours, after that $1 \mathrm{ml}$ of nutrient broth was added to each plate, the growth harvested then transferred to small vials containing one $\mathrm{ml}$ of sterilized $80 \%$ glycerol then stored at $-20^{\circ} \mathrm{C}$ [15].

\section{III.1 Specimen collection}

\section{Results and Discussion}

A total of 45 samples were collected from the nasal cavity of biology and medical microbiology students that they were taking education in the 2012-2013 at Koya University, Faculty of Science and health and also 35 samples were collected from the nasal cavity of healthy worker that work in Mas cement factory in the (2012-2013). The samples were arrived to the laboratory and planted on the special media then after 24 hours characteristics of isolates were studied, through culturing them on differential medium, such as blood agar and macConkey agar, chocolate agar. The results of the students showed seventeen of the students that attended in this research have no growth isolated. Sixteen $(35.55 \%)$ of them were found out that they had Streptococcus spp. and nine (20\%) of them have Staphylococcus spp , two (4.44\%) of them had E. coli and two (4.44\%) of the students had Klebsiella spp. as demonstrate in table (1) while the results of the cement workers were attended in this research two of the workers had no growth isolated, eighteen $(51.77 \%)$ of the workers were found that they have Moraxella catarrhalis, while thirteen (37.14\%) of the workers had Candida albicans, five of them $(14.28 \%)$ had streptococcus faecalis, three $(8.57 \%)$ of them were found that they had E.coli, and two (5.71\%) of them had Klebsiella spp., and one (2.85\%) of the growth found Citrobacter freundii as demonstrate in table (2).

Table (1) results of nose swab culture among students at Koya University.

\begin{tabular}{|l|l|l|}
\hline No. & Sample No. & Growth \\
\hline 1 & $1,3,5,7,9,12,14,23,24,25,27,29,30,31,40,44,45$ & No growth isolated \\
\hline 2 & $4,8,13,17,18,19,20,22,26,34,35,37,38,39,42,43$ & Streptococcus spp. \\
\hline 3 & $2,11,15,16,21,32,33,36,41$ & Staphylococcus spp. \\
\hline 4 & 6,28 & Klebsiella spp \\
\hline 5 & 10,15 & E. coli \\
\hline
\end{tabular}


A comparative study on detecting bacterial flora of the nasal cavity in normal healthy workers ....

Table (2) results of nose swab culture among healthy workers that they work in cement factory in sulaimanya city

\begin{tabular}{|l|l|l|}
\hline No. & \multicolumn{1}{|c|}{ Samples No. } & \multicolumn{1}{c|}{ Growth } \\
\hline 1 & 34,35 & N.G \\
\hline 2 & $\begin{array}{l}1,3,5,6,8,10,11,15,16,18,20 \\
22,23,26,27,28,29,30\end{array}$ & Moraxella catarrhalis \\
\hline 3 & $3,5,6,8,9,17,19,22,28,29,30,31,32$ & Candida albicans \\
\hline 4 & $6,18,24,26,28$ & streptococcus faecalis \\
\hline 5 & $4,14,15$ & E.coli \\
\hline 6 & 10,24 & Klebsiella spp \\
\hline 7 & 9 & Citrobacter freundii \\
\hline
\end{tabular}

III.4 Isolation and Identification of microorganisms that obtained from nasal cavity of Koya University students

After collection, isolated microorganisms and the characteristics of isolates were studied, through culturing them on differential medium, such as MacConkey agar, blood agar, chocolate agar and manitol salt agar.

The Identification and biochemical test for bacterial isolates:

Forty five students were attending in this research at Koya University, seventeen $(37.77 \%)$ samples had no growth isolated and sixteen students (35.55\%) were the nasal streptococcus spp. Carrier as shown in table (1).

\section{III.4.1Streptococcus agalactiae:}

$2.22 \%$ (one student) was the nasal Streptococcus agalactiae carrier overall forty five students as shown in figure (5). This species is gram positive coccus, arranged in pairs and chains. Colony morphology on blood agar, Grayish-white, mucoid, creamy, narrow zone of b-hemolytic colonies that is larger than colonies of other B-hemolytic streptococci as show in "fig 1"

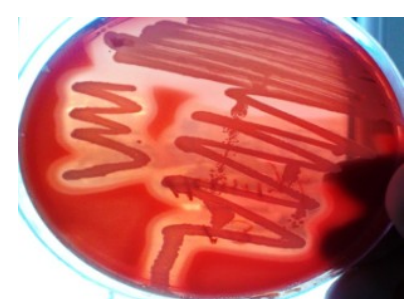

Figure (1) Colonies of Streptococcus agalactiae on blood agar exhibiting beta (clear) hemolysis.

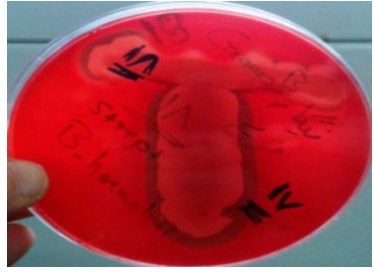

Figure (2) a- CAMP factor test

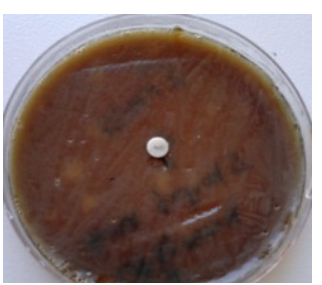

b- bacitracin activity negative

Tests of value for identification of $\mathrm{S}$. agalactiae include negative for bile esculin hydrolysis, bacitracinresistant as show in figure (2b). Positive for production of CAMP factor as shown in figure (2a), catalase negative, as mentioned before by [16].

\section{III.4.2 Streptococcus faecalis}

Two of the students (4.44\%) and five of the workers $(14.28 \%)$ were the nasal streptococcus faecalis Carrier as shown in table (1) and (2), were the nasal streptococcus faecalis Carrier. this bacteria is nonhemolytic streptococci, enterococcus, group D streptococci gamma haemolytic on blood agar and the colonies appear white and grow on maCchonky agar appear small pink colony,optimal temperature for growth of Enterococcus faecalis is $35^{\circ} \mathrm{C}$ [17].

Biochemical test also done: ferment glucose without gas production, bile esculin hydrolysis ability to grow in $40 \%$ bile and hydrolyze esculin are features of streptococci that possess group d antigen as shown in "fig 3", non motile in semisolid manitol, and catalase negative. Microscopic character Gram- positive cocci, arranged individually, in pairs, or short chains [17]. 


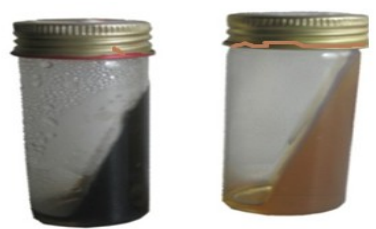

Figure (3) esculin hydrolysis test

\section{III.4.3 Streptococcus pyogenes}

From forty five student's three students $6.66 \%$ was the nasal Streptococcus pyogenes carrier as shown in "fig 5", this species of streptococcus belong to group A according Rebecca Lancefield. When the bacteria are grown on agar that contains sheep blood, it will appear as a translucent colony with a zone of hemolysis surrounding the colony. This is due to specific enzymes produced by this type of streptococcus, producing $\beta$ hemolysis (a clear zone of hemolysis with no color change) on blood agar, sensitive to bacitracin disk ( $10 \mathrm{u}$ ) as shown in figure (4a) (resist to trimethoprem (SXT) and negative to CAMP factor. Microscopically, Streptococcus pyogenes is gram-positive cocci or appears in chain as previously regards [18].

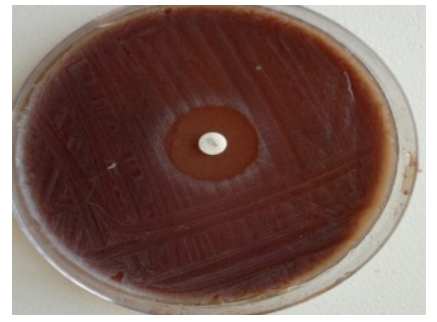

Figure (4) group A streptococcus is susceptible to bacitracin disk

Nine students also had other species of streptococci as demonstrate in "fig 5", non hemolytic or they have gamma hemolytic on blood agar on MaCconky agar no growth appear, microscopic character Grampositive cocci, arranged individually, in pair or chain.

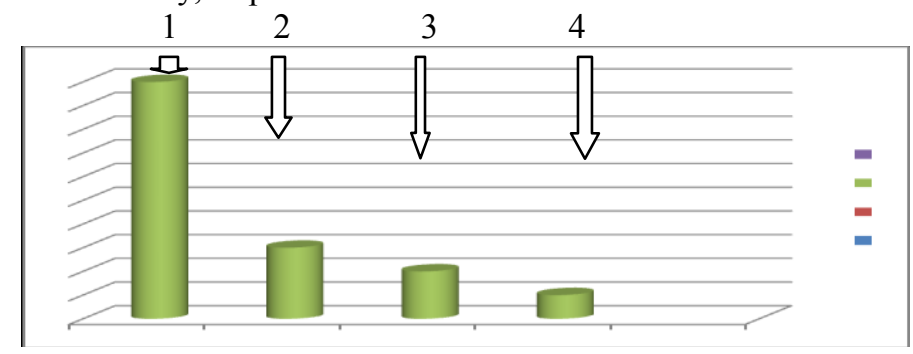

Figure (5) nasal Streptococcus spp. carriage according to distribution of the species among students in Koya university $\{1-(1-10)$ of the isolates other Streptococcus spp. 2-three of the isolates were Streptococcus pyogenes 3-two of the isolates were streptococcus faecalis 4-one of the isolate was Streptococcus agalactiae\}.

\section{III.4.4 Staphylococcus spp.}

After isolation and identification appear nine students (20\%) were the nasal Staphylococcus spp. Carrier as shown in table (1).

Five (55.55\%) of students had the nasal Staph epidermis carrier among the students attending in this study as shown in "fig 6 "

Four (44.44 \%) of the students were the nasal Staph aureus carrier as demonstrate in "fig 6".

\section{III.4.4.1 Staph aureus}

The nose swab samples taken from students were examined through microbiological methods. On blood agar Staph aureus colony morphology on blood agar appears round, convex, mucoid, and adherent to the agar and yellow in color. Positive for catalase, Oxidase negative and are Voges-Proskauer positive [13] All pathogenic strains of S. aureus are coagulase positive. In general microscopically they are G+ve cocci, in cluster, non motile, non capsulate, non spore forming [20]. 


\section{III.4.4.2 Staphylococcus epidermidis}

After isolation and identification appear gram positive cocci in clusters as shown in "fig 7" facultative anaerobe, Non motile not spore forming do not have capsule [20].
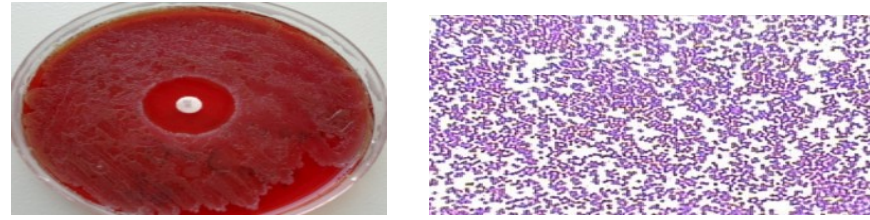

Figure (7) suceptability test to novobiocine disk for differentiate between Staphylococcus Epidemidis and Staphylococcus saprophyticus and gram stain morphology of Staphylococci

\section{III.4.4.3 Klebsiella spp}

According to smear preparation by Gram stain the bacterial cells are gram negative, rods, short, non motile[21], [10].

biochemical test for bacterial isolates: not produce indole from tryptophan it mean that the result is negative, also negative for methyl red, positive for catalase, vogs proskaure and citrate, TSI fermentation glucose \& lactose with producing gas, but it was negative for oxidase, and negative for $\mathrm{H} 2 \mathrm{~S}$ production and urease production [13]. Thus two of the students had Klebsiella spp. and two (4.44\%) of the workers that work in mas cement factory had Klebsiella spp.

\section{III.4.4.4 Escherichia coli}

Two of the students $(4.44 \%)$ and three of the workers $(8.57 \%)$ that attending in this research had Escherichia coli carrier as shown in table (1) and (2). The characteristics of the isolates were studied, through culturing them on differential media, such as macConkey agar. The isolates appear smooth and circular, and pink in color by fermenting lactose, on EMB media the isolates are highly pigmented and have small smooth metallic sheen colonies asshow in "fig 8 ".

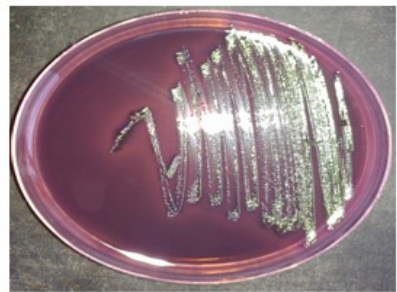

Figure (8) E. coli isolate on EMB agar

The biochemical test for bacterial isolates of the bacteria produce indole from tryptophan it mean s that the result is positive, also positive for methyl red, catalase, semisolid manitole ( fermentation manitole \& motility) and TSI fermentation glucose\& lactose with producing gas, but it was negative for (oxidase, citrate utilization, vogas proskaur, gelatine liquefication $\mathrm{H} 2 \mathrm{~S}$ production and urease production)[13].

The results show in this study the Streptococcus spp. carriage was meaningfully higher than other bacterial species among the students $35.55 \%$ including (Streptococcus progenies, Streptococcus agalactiae, Streptococcus faecalies), after that $20 \%$ of Staphylococcus spp. colonized among students including (Staphylococcus aureus and Staphylococcus epidermidis) and (4.44\%) for both of Klebsiella spp. and Escherichia coli as demonstrate in "fig 9".

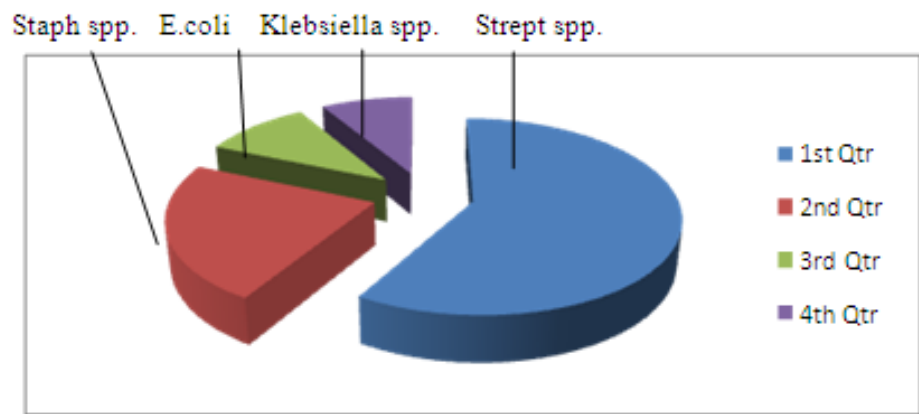

Figure (9) rate of bacterial species among students 
A comparative study on detecting bacterial flora of the nasal cavity in normal healthy workers ....

III.5 Thirty five healthy cement workers were attending in this research two $(5.71 \%)$ of them have no growth isolated and eighteen (51.42\%) were the nasal Moraxella catarrhalis Carrier as shown in table (2).

\section{III.5.1 Moraxella catarrhalis}

Thirty five workers attending the research were in cement factory, eighteen of the workers were found out that they have Moraxella catarrhalis as shown in table (2), Moraxella catarrhalis the most frequent distributed bacterial isolate among the workers as shown in "fig 10" Members of the genus microscopically; Moraxella are nonmotile, gram-negative coccobacilli, non spore forming that are generally found in pairs, as show in "fig 11 ".

Moraxella are aerobic, on blood agar appear white smooth colony, and do not grow on MaCchonky agar it means it is not tolerate bile salt.

Biochemical test: oxidase-positive, catalase positive, fastidious organisms that M. catarrhalis is an opportunistic pulmonary invader, and causes harm especially in patients who have compromised immune systems or any underlying chronic disease, these bacteria are known to cause otitis media, bronchitis, sinusitis, and laryngitis. [22]. Elderly patients and long-term heavy smokers with chronic pulmonary disease should be aware that M. catarrhalis is associated with bronchopneumonia, as well as exacerbations of existing chronic obstructive pulmonary disease (COPD) [23].

\section{$\begin{array}{llllll}1 & 2 & 3 & 4 & 5 & 6\end{array}$}

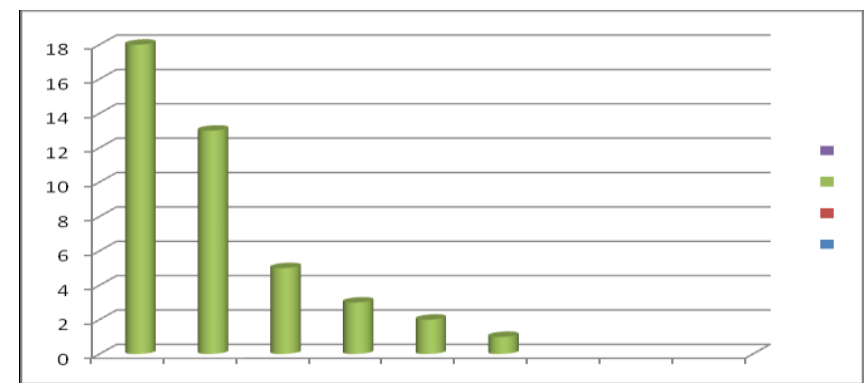

Figure (10) rate of microorganisms that distributed among works, 1-Moraxella catarrhalis eighteen (51.42\%) 2- Candida albicans (37.14\%) 3- streptococcus faecalis(14.28\%) 3- E. coli (8.57\%) 4Klebsiella spp (5.71\%) 5-Citrobacter freundii (2.85\%)

"Fig 10" rate of microorganisms that distributed among works, 1-Moraxella catarrhalis eighteen (51.42\%) 2Candida albicans (37.14\%) 3- streptococcus faecalis (14.28\%) 3- E. coli (8.57\%) 4- Klebsiella spp (5.71\%) 5- Citrobacter freundii $(2.85 \%)$.
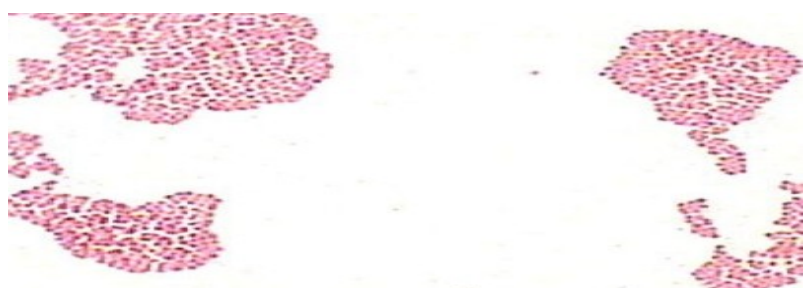

Figure (11) gram stains character for Moraxella catarrhalis.

\section{III.5.2 Candida albican}

Thirty five workers attending the research were in cement factory, thirteen of the workers were found that they have Candida albican as show in table (2).

After collection and isolated microorganisms the characteristics of isolates were studied, through culturing them on blood agar, the isolates appear white smooth colony as shown in figure (2), Candida is gram positive, and it grows overnight on most bacterial and fungal media.

Candida produces germ tubes and pseudohyphae may be formed from budding yeast cells that remain attached to each other. Spores may be formed on the pseudomycelium. These are called chlamydospores and they can be used to identify different species of Candida [24]. The Candida albican being an important pathogen is seen in cement factory workers by $(37.14 \%)$ as shown in "fig 12 ". 


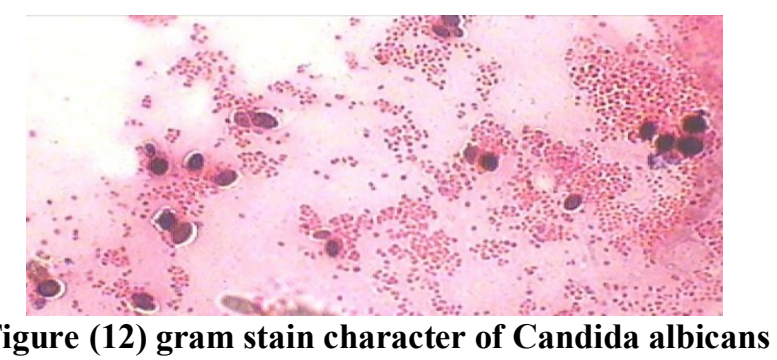

\section{III.5.3 Citrobacter freundii}

After isolation the characteristics of isolates were studied, through culturing them on differential medium, such as MacConkey agar., this isolate appear smooth and circular, and pink in color by fermenting lactose on MacConkey agar, C .amalonaticus, C. koseri, and C. freundii use solely citrate as a carbon source. Citrobacter species are differentiated by their ability to convert tryptophan to indole, ferment lactose, and utilize malonate and Citrobacter freundii on TSI fermentation glucose\& lactose with producing gas and H2S, catalase positive, Late or weak urease producers, oxidase negative. Citrobacter is a genus of Gram-negative coliform bacteria in the Enterobacteriaceae family [25]. In this study one strain of Citrobacter freundii isolated from worker's nose cavity $(2.85 \%)$ as shown in table (2).

The results show in this study the Moraxella catarrhalis carriage was significantly higher than other bacterial species among the workers (51.77\%), after that (37.14\%) Candida albicans colonized among the workers and some of workers were nasal (streptococcus faecalis, E.coli, Klebsiella spp., Citrobacter freundii carrier and the percentage found $(14.28 \%, 8.57 \%, 5.71 \%$, and $2.85 \%)$ respectively as shown in "fig 11 ".

These results indicate the workers that work in cement factory might have been contaminated with above microorganisms that they being an important pathogens, while among the students of Faculty of Science and Health from Koya University. The nasal Streptococcus Isolates were found out meaningfully that was higher than other isolates.

Microorganisms distributed among workers, Moraxella catarrhalis, Candida albicans, streptococcus faecalis, E. coli, Klebsiella spp and Citrobacter freundii.

This study has provided evidence that the workers exposed to large concentrations of cement agree with some previous studies have examined the effect of such levels of exposure to cement and shown the potential for allergic respiratory ill health, as show in the study [3]. Microbiological activity is fundamental to the composting process; therefore any handling of composting material is likely to make airborne significant quantities of those micro-organisms (referred to as bioaerosols). Workers mechanically handling compost on these sites may therefore be at risk of considerable exposure to bioaerosols depending on their work task, their proximity to the bioaerosol source and the control measures put in place. In addition, because the work is largely done out of doors, there is the potential for bioaerosols generated to disperse some distance from the point source. Consequently, there is concern that people living or working in the vicinity of waste composting sites (sensitive receptors) may also be exposed to these aerosols.

Also the results indicate that Faculty of Science and Health students might have been contaminated with (Streptococcus pyogenes and other species of streptococcus and Staphylococcus aureus), during laboratory practice. That this two species being an important pathogen is seen in biology and medical microbiology students taking education in (2012-2013), the results agree with[6], that show The nasal MRSA carriage was investigated in Nebraska High School athletes between 2006-2008 years and the community acquired MRSA (CMRSA) was isolated in 51 out of 271 students. That MRSA carriage is seen in this ratio in healthy individuals not taking place in the hospital medium shows dimensions of the risk to be met with respect to the public health [26].

[6] BHS carriage was also investigated in midwifery students, the Group A BHS carriage was available in $1.5 \%$ of students and there wasn't meaningful difference according to groups. This situation can be dependent on that BHS is not a pathogen acquired in the hospital medium and it is generally a community acquired factor. Since the A group leads to diseases such as hemolytic streptococci and streptococcal tonsillopharyngitis, it is very important especially for the child health. These bacteria can infect individuals in the places where the populations such as school, day nursery and hostel are intensive through aerogenic way [27].

\section{Conclusion}

The results indicate that Faculty of Science and Health students might have been contaminated with (Streptococcus pyogenes and other species of streptococcus and Staphylococcus aureus, Klebsiella spp and E. coli).

The results indicate that the workers in cement factory that expose with the aerosol and have contact with cement directly might have been contaminated with (Moraxella catarrhalis, Candida albicans, 
streptococcus faecalis, E.coli,and Klebsiella spp. These species being an important pathogen that distribute among workers especially with those have immune compromise system.

The results indicate that the environment around the workers and around the students two different environment because the results show two different types of microorganisms contaminated these two groups.

\section{Recommendations}

Microbiological scanning must be done to the infected workers and students.

The carriers must be determined and treated. The hands must be washed before and after the contact with the cement and with any laboratory devices a suitable sapray must be applied. Contaminated material must be disinfected or sterilized and the gloves and aprons must be used.

This study has provided evidence of the potential for compost site workers to be exposed to large concentrations of cement, and some previous studies have examined the effect of such levels of exposure to cement and shown the potential for allergic respiratory ill health.

\section{References}

[1]. D.N. Frank,.; L.M. Feazel; M.T. Bessesen; C.S. Price and E.N. Janoff. The Human Nasal Microbiota and Staphylococcus aureus Carriage. PLoS ONE 5(5): e10598. doi:10.1371/journal.pone.0010598), 2010.

[2]. T. Rasmussen, L. P. Kirkeby, K. Poulsen, J. Reinholdt and M. Kilian, Resident aerobic microbiota of the adult human nasal cavity, APMIS. US National Library of Medicine National Institutes of Health, 2008 108(10):663-75.

[3]. A. Stephen Morse, Medical Microbiology. 4th edition. Chapter 14Neisseria, Moraxella, Kingella and Eikenella, Bookshelf ID: NBK7650PMID: 21413257, 2010.

[4]. S. Baron, Medical Microbiology. 4th edition. Chapter 6 (Normal Flora Bookshelf ID: NBK7617 PMID: 21413249, 1996.

[5]. H. Ucuncu., H. Uslu, A. Ozbek, B. Aktan, Y. Sutbeyaz, and E. Altas, Comparison of the bacterial flora of the nasal vestibule and cavity in haemodialysis patients, 29(5), 2009,251-2,4.

[6]. K. Ekrem.; A. Ozer; M. Aral, and M. Miraloglu. A Research of nasal methicillin resistant/sensitive Staphylococcus aureus and pharyngeal beta-haemolytic Streptococcus carriage in midwifery students in Kahramanmaras, Eastern Mediterranean Region of Turkey. Kocatepe Medical Journal 14: 77-82/Mayis 2013.

[7]. S . Stephen, B. Alison, K. Adrian \& C. Brian, Bioaerosol emissions from waste composting and the potential for workers' exposure, Health and Safety Executive. Harpur Hill Buxton Derbyshire SK17 9JN RR786, Research Report, RR786 Research Report, 2010,2.

[8]. J. O. Hendley, F. G. Hayden \& B.Winther, Weekly point prevalence of Streptococcus pneumoniae, Haemophilus influenzae and Moraxella catarrhalis in the upper airways of normal young children: effect of respiratory illness and season. APMIS 113, 2005, 213-220.

[9]. L. Dougherty., and S. Lister. The Royal Marsden Hospital Manual of Clinical Nursing Procedures (6th ed). Oxford: Blackwell Publishing (2004).

[10]. K. J. Ryan, and C.G. RaySherris, Medical Microbiology (4th ed.). McGraw Hill. ISBN 0-8385-8529-9, 2004.

[11]. R. Jackie., BIOL2420., 2011 delrio.dcccd.edu/jreynolds/microbiology/2420/files/Streptococci.pdf

[12]. C. Harvey; A. Richard.; C. Pamela.; Fisher, and D. Bruce., Lippincott's Illustrated Reviews: Microbiology, 2nd Edition Lippincott Williams \& Wilkins, 2007.

[13]. Koneman's M.D., Color Atlas and Textbook of "Diagnostic Microbiology", 2006. http://www.livestrong.com/article/83622-characteristics-staphylococcus aureusixzz2PZfT15eW.

[14]. W. Levinson, and E. Jawetz, Medical microbiology and immunology. Hall International. Six edd. Lange Medical Books/McGrawHill. Medical Publishing . New York., 2000.

[15]. Colour Atlas, R. M. Practical Pathology and Microbiology.Mosby-year Book,Inc., U.S.A. 2006.

[16]. M. R Wesswls and D. L. kasper, infectious disease, $3^{\text {rd }}$ Ed. Philadelphia : Lippincott Williams\&wilkins, $2004,444$.

[17]. S.G. Amyes "Enterococci and streptococci". Int. J. Antimicrob. Agents. 29 Suppl 3: S43-52. doi:10.1016/S0924-8579(07)721775. PMID 17659211, 2007.

[18]. Kenneth Todar,( 2011) - www.textbookofbacteriology.net

[19]. A. H. Richard, C. C. Pamela, and D. Bruce, Microbiology $2^{\text {nd }}$ edition Lippincott"s illustrated Review, 2000.

[20]. W. Levinson, and E. Jawetz., Medical microbiology and immunology, Hall International. Six edd. Lange Medical Books/McGrawHill. Medical Publishing. New York, 2000.

[21]. F. Mawas, HoMM, M. J. Corbel "Current progress with Moraxella catarrhalis antigens as vaccine candidates". Expert Rev Vaccines 8 (1): 77 90. doi:10.1586/14760584.8.1.77.PMID 19093775, 2009.

[22]. Melendez, R. Philipp and H. j. Royce, "Bacteremia and Septic Arthritis Caused by Moraxella catarrhalis." Reviews of Infectious Diseases 13, no. 3, 1991, 428-429

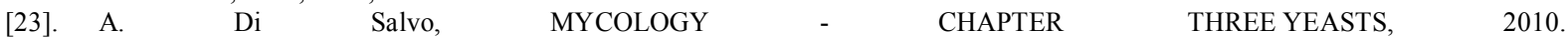
http://www.wrongdiagnosis.com/mistakes/nosocomial.htm?ktrack=kcplink

[24]. J.T. Wang., S.C. Chang; Y.C. Chen, and K.T. Luh. Comparison of antimicrobial susceptibility of Citrobacter freundii isolates in two different time periods.” The Journal of Microbiology, Immunology and Infection. Dec; 33(4): 2000, 258-62.

[25]. F. Bryan, M.P.H. Buss, W. Shawn, R.N. Mueller, M. Theis, A. Keyser. et al.. Population-Based Estimates of Methicillin-Resistant Staphylococcus aureus (MRSA) Infections Among High School Athletes-Nebraska; 25(4):282-91. 2009.

[26]. D. J. Weber, W. A. Rutala, F. W. Denny. Management of healthcare workers with pharyngitis or suspected streptococcal infections. Infect Control Hosp Epidemiol, 1996, 17:753-61. 